Otterbein University

Digital Commons @ Otterbein

Physics Faculty Scholarship

Physics

10-23-2014

\title{
Higgs Boson Mass in the Standard Model at Two-Loop Order and Beyond
}

Steve P. Martin

Fermilab \& Northern Illinois University

David G. Robertson

Otterbein University

Follow this and additional works at: https://digitalcommons.otterbein.edu/phys_fac

Part of the Physics Commons

\section{Repository Citation}

Martin, Steve P. and Robertson, David G., "Higgs Boson Mass in the Standard Model at Two-Loop Order and Beyond" (2014). Physics Faculty Scholarship. 18.

https://digitalcommons.otterbein.edu/phys_fac/18

This Article is brought to you for free and open access by the Physics at Digital Commons @ Otterbein. It has been accepted for inclusion in Physics Faculty Scholarship by an authorized administrator of Digital Commons@ Otterbein. For more information, please contact digitalcommons07@otterbein.edu. 


\title{
Higgs boson mass in the standard model at two-loop order and beyond
}

\author{
Stephen P. Martin ${ }^{1,2}$ and David G. Robertson ${ }^{3}$ \\ ${ }^{1}$ Department of Physics, Northern Illinois University, DeKalb, Illinois 60115, USA \\ ${ }^{2}$ Fermi National Accelerator Laboratory, P.O. Box 500, Batavia, Illinois 60510, USA \\ ${ }^{3}$ Department of Physics, Otterbein University, Westerville, Ohio 43081, USA
}

(Received 20 July 2014; published 23 October 2014)

\begin{abstract}
We calculate the mass of the Higgs boson in the standard model in terms of the underlying Lagrangian parameters at complete 2-loop order with leading 3-loop corrections. A computer program implementing the results is provided. The program also computes and minimizes the standard model effective potential in Landau gauge at 2-loop order with leading 3-loop corrections.
\end{abstract}

DOI: 10.1103/PhysRevD.90.073010

PACS numbers: $14.80 . \mathrm{Bn}$

\section{INTRODUCTION}

The Large Hadron Collider (LHC) has discovered [1] a Higgs scalar boson $h$ with mass $M_{h}$ near $125.5 \mathrm{GeV}$ [2] and properties consistent with the predictions of the minimal standard model. At the present time, there are no signals or hints of other new elementary particles. In the case of supersymmetry, the limits on strongly interacting superpartners are model dependent, but typically extend to over an order of magnitude above $M_{h}$. It is therefore quite possible, if not likely, that the standard model with a minimal Higgs sector exists as an effective theory below $1 \mathrm{TeV}$, with all other fundamental physics decoupled from it to a very good approximation. Within this model, precision calculations can help to relate observable quantities to the underlying Lagrangian parameters, as well as help to constrain new physics models, including those for which decoupling may not hold.

One such observable quantity is the physical mass $M_{h}$ itself. At tree level, $M_{h}$ is directly proportional to the square root of the Higgs field self-interaction coupling, $\lambda$. One important question has to do with the stability of the standard model vacuum [3-14]. The observed value of $M_{h}$ is in the range that would apparently correspond to metastability of the vacuum [15-19], assuming that there is no new physics between the electroweak scale and the Planck scale. It is therefore important to pin down the relationship between $\lambda$ and $M_{h}$ as accurately as possible. Parametric uncertainties, notably the dependences on the top-quark mass and the QCD coupling, are not insignificant, and will likely remain so for some time. However, our attitude is that theoretical calculations should, to the extent possible, be pushed to the point that all limitations of our understanding can be reliably and unambiguously blamed on experimental error.

The purpose of this paper is to present a full 2-loop calculation of the minimal standard model Higgs boson pole mass $M_{h}$, in terms of the $\overline{\mathrm{MS}}$ Lagrangian parameters $v, \lambda$, $y_{t}, g, g^{\prime}, g_{3}$, with the leading 3-loop corrections in the limit $g_{3}, y_{t} \gg \lambda, g, g^{\prime}$. The relations between these parameters and other observables, such as the physical masses of the top quark and the $Z$ and $W$ bosons, are left to separate calculations. The result for $M_{h}$ is probably too long to present as an analytical formula in print without forfeiting the goodwill of the reader, and in any case evaluation of it will necessarily rely on numerical work done by computer. We therefore present most of our results in the form of an electronic file, and as a public computer code. The computer code also performs the related task of minimizing the 2-loop effective potential [20] of the standard model with leading 3-loop corrections [21], implementing the form of the minimization condition given recently in [22,23], which resummed Goldstone contributions to eliminate spurious imaginary parts and potentially infrared singular contributions.

Our calculation is restricted to Landau gauge, but in general the complex pole mass [24-28] in quantum field theory is a physical observable and is therefore expected to be both renormalization group invariant and independent of the gauge fixing parameters. The latter property has been shown to all orders in perturbation theory for the particles of the standard model in Ref. [29]. Because our calculation is restricted to Landau gauge, the gauge invariance cannot serve as a check of its correctness, but numerous other checks will be described below.

Previous work on the 2-loop contributions to the relation between $\lambda$ and $M_{h}$ includes the QCD corrections [17,18], which can be obtained from the 2-loop QCD correction $[30,31]$ to the Higgs self-energy function. The non-QCD corrections have been obtained by [18] and [19] but were given there only in the form of simple interpolating formulas.

\section{HIGGS POLE MASS AT 2-LOOP ORDER}

To fix our conventions and notation, we write the Higgs kinetic and self-interaction Lagrangian as

$$
\mathcal{L}=-\partial^{\mu} \Phi^{\dagger} \partial_{\mu} \Phi-\Lambda-m^{2} \Phi^{\dagger} \Phi-\lambda\left(\Phi^{\dagger} \Phi\right)^{2},
$$

where we use the metric with signature $(-,+,+,+)$, and $m^{2}<0$, and the complex doublet Higgs field is 


$$
\Phi(x)=\left(\begin{array}{c}
\frac{1}{\sqrt{2}}\left[v+h(x)+i G^{0}(x)\right] \\
G^{+}(x)
\end{array}\right) .
$$

Here $v$ is the Higgs vacuum expectation value (VEV), which we take to be evaluated at the minimum of the effective potential evaluated at 2-loop order with leading 3-loop corrections. This means that the sum of tadpole diagrams (including the tree-level one) vanishes at that same order, and so need not be included. Because the Landau gauge is used for the evaluation of the effective potential in [20-23], our calculation also is restricted to that gauge-fixing scheme.

The other relevant couplings in the theory are the topquark Yukawa coupling $y_{t}$ and the $S U(3)_{c} \times S U(2)_{L} \times$ $U(1)_{Y}$ gauge couplings $g_{3}, g, g^{\prime}$. In principle, the bottom quark and other fermion Yukawa couplings can also be included, but they make only a very tiny difference even at 1-loop order, where their inclusion is straightforward (see below). All of the couplings $\lambda, m^{2}, y_{t}, g_{3}, g, g^{\prime}$, and the VEV $v$, are running parameters in the $\overline{\mathrm{MS}}$ scheme.

In order to obtain the Higgs boson physical mass $M_{h}$, we calculate the self-energy function

$$
\Pi(s)=\frac{1}{16 \pi^{2}} \Pi^{(1)}(s)+\frac{1}{\left(16 \pi^{2}\right)^{2}} \Pi^{(2)}(s)+\cdots
$$

consisting of the sum of all 1-particle-irreducible 2-point Feynman diagrams, in the regulated theory in $d=4-2 \epsilon$ dimensions. In this paper, factors of $1 /\left(16 \pi^{2}\right)^{\ell}$ are extracted as a way of signifying the loop order $\ell$. Rather than including counterterm diagrams separately, we found it more efficient to do the calculation in terms of the bare quantities $\lambda_{B}, m_{B}^{2}, y_{t B}, g_{3 B}, g_{B}, g_{B}^{\prime}$, and VEV $v_{B}$, and then reexpress the results in terms of the $\overline{\mathrm{MS}}$ quantities. The complex pole squared mass is the solution of

$$
\begin{aligned}
M_{h}^{2}-i \Gamma_{h} M_{h} \equiv s_{\text {pole }}= & m_{B}^{2}+3 \lambda_{B} v_{B}^{2}+\frac{1}{16 \pi^{2}} \Pi^{(1)}\left(s_{\text {pole }}\right) \\
& +\frac{1}{\left(16 \pi^{2}\right)^{2}} \Pi^{(2)}\left(s_{\text {pole }}\right),
\end{aligned}
$$

where 3-loop order effects are consistently neglected in this section. We then apply the $\overline{\mathrm{MS}}$ relations between bare and renormalized parameters:

$v_{B}^{2}=\mu^{-2 \epsilon} v^{2}\left[1+\frac{1}{16 \pi^{2}} \frac{c_{1,1}^{\phi}}{\epsilon}+\frac{1}{\left(16 \pi^{2}\right)^{2}}\left(\frac{c_{2,2}^{\phi}}{\epsilon^{2}}+\frac{c_{2,1}^{\phi}}{\epsilon}\right)+\cdots\right]$,

$\lambda_{B}=\mu^{2 \epsilon}\left[\lambda+\frac{1}{16 \pi^{2}} \frac{c_{1,1}^{\lambda}}{\epsilon}+\frac{1}{\left(16 \pi^{2}\right)^{2}}\left(\frac{c_{2,2}^{\lambda}}{\epsilon^{2}}+\frac{c_{2,1}^{\lambda}}{\epsilon}\right)+\cdots\right]$,

$$
m_{B}^{2}=m^{2}+\frac{1}{16 \pi^{2}} \frac{c_{1,1}^{m^{2}}}{\epsilon}+\frac{1}{\left(16 \pi^{2}\right)^{2}}\left(\frac{c_{2,2}^{m^{2}}}{\epsilon^{2}}+\frac{c_{2,1}^{m^{2}}}{\epsilon}\right)+\cdots,
$$

$$
\begin{gathered}
y_{t B}=\mu^{\epsilon}\left[y_{t}+\frac{1}{16 \pi^{2}} \frac{c_{1,1}^{y_{t}}}{\epsilon}+\cdots\right], \\
g_{B}=\mu^{\epsilon}\left[g+\frac{1}{16 \pi^{2}} \frac{c_{1,1}^{g}}{\epsilon}+\cdots\right], \\
g_{B}^{\prime}=\mu^{\epsilon}\left[g^{\prime}+\frac{1}{16 \pi^{2}} \frac{c_{1,1}^{g^{\prime}}}{\epsilon}+\cdots\right], \\
g_{3 B}=\mu^{\epsilon}\left[g_{3}+\cdots\right],
\end{gathered}
$$

to obtain $s_{\text {pole }}$ in terms of the renormalized parameters. Here $\mu$ is the regularization scale, related to the $\overline{\mathrm{MS}}$ renormalization scale $Q$ by

$$
Q^{2}=4 \pi e^{-\gamma_{E}} \mu^{2},
$$

where $\gamma_{E}=0.5772 \ldots$ is the Euler-Mascheroni constant, and the counterterm coefficients are, to the orders required for this paper:

$$
c_{1,1}^{\phi}=-3 y_{t}^{2}+\frac{9}{4} g^{2}+\frac{3}{4} g^{\prime 2},
$$

$$
\begin{aligned}
c_{2,2}^{\phi}= & 12 g_{3}^{2} y_{t}^{2}-\frac{9}{4} y_{t}^{4}-\frac{27}{8} y_{t}^{2} g^{2}-\frac{1}{8} y_{t}^{2} g^{\prime 2}-\frac{33}{32} g^{4} \\
& +\frac{27}{16} g^{2} g^{\prime 2}+\frac{91}{32} g^{\prime 4},
\end{aligned}
$$

$$
\begin{aligned}
c_{2,1}^{\phi}= & -10 g_{3}^{2} y_{t}^{2}+\frac{27}{8} y_{t}^{4}-\frac{45}{16} y_{t}^{2} g^{2}-\frac{85}{48} y_{t}^{2} g^{\prime 2}+\frac{271}{64} g^{4} \\
& -\frac{9}{32} g^{2} g^{\prime 2}-\frac{431}{192} g^{\prime 4}-3 \lambda^{2}, \\
c_{1,1}^{\lambda}= & -3 y_{t}^{4}+6 \lambda y_{t}^{2}+12 \lambda^{2}-\frac{9}{2} \lambda g^{2}-\frac{3}{2} \lambda g^{\prime 2}+\frac{9}{16} g^{4} \\
& +\frac{3}{8} g^{2} g^{\prime 2}+\frac{3}{16} g^{\prime 4}, \\
c_{2,2}^{\lambda}= & 24 g_{3}^{2} y_{t}^{4}-24 g_{3}^{2} y_{t}^{2} \lambda-\frac{45}{2} y_{t}^{6}+\frac{27}{2} y_{t}^{4} g^{2}+\frac{13}{2} y_{t}^{4} g^{\prime 2} \\
& -\frac{9}{2} y_{t}^{4} \lambda+108 y_{t}^{2} \lambda^{2}-\frac{135}{4} y_{t}^{2} \lambda g^{2}-\frac{53}{4} y_{t}^{2} \lambda g^{\prime 2}+\frac{27}{16} y_{t}^{2} g^{4} \\
& +\frac{9}{8} y_{t}^{2} g^{2} g^{\prime 2}+\frac{9}{16} y_{t}^{2} g^{\prime 4}+144 \lambda^{3}-81 \lambda^{2} g^{2}-27 \lambda^{2} g^{\prime 2} \\
& +24 \lambda g^{4}+\frac{45}{4} \lambda g^{2} g^{\prime 2}-\frac{7}{4} \lambda g^{4}-\frac{195}{64} g^{6}-\frac{119}{64} g^{4} g^{\prime 2} \\
& +\frac{37}{64} g^{2} g^{\prime 4}+\frac{73}{64} g^{\prime 6},
\end{aligned}
$$




$$
\begin{aligned}
c_{2,1}^{\lambda}= & -8 g_{3}^{2} y_{t}^{4}+20 g_{3}^{2} y_{t}^{2} \lambda+\frac{15}{2} y_{t}^{6}-\frac{2}{3} y_{t}^{4} g^{\prime 2}-\frac{3}{4} y_{t}^{4} \lambda-36 y_{t}^{2} \lambda^{2} \\
& +\frac{45}{8} y_{t}^{2} \lambda g^{2}+\frac{85}{24} y_{t}^{2} \lambda g^{\prime 2}-\frac{9}{16} y_{t}^{2} g^{4}+\frac{21}{8} y_{t}^{2} g^{2} g^{\prime 2} \\
& -\frac{19}{16} y_{t}^{2} g^{\prime 4}-78 \lambda^{3}+27 \lambda^{2} g^{2}+9 \lambda^{2} g^{\prime 2} \\
& -\frac{73}{32} \lambda g^{4}+\frac{39}{16} \lambda g^{2} g^{2}+\frac{629}{96} \lambda g^{4}+\frac{305}{64} g^{6}-\frac{289}{192} g^{4} g^{\prime 2} \\
& -\frac{559}{192} g^{2} g^{\prime 4}-\frac{379}{192} g^{\prime 6}, \\
c_{1,1}^{m^{2}}= & m^{2}\left[3 y_{t}^{2}+6 \lambda-\frac{9}{4} g^{2}-\frac{3}{4} g^{\prime 2}\right]
\end{aligned}
$$$$
c_{2,2}^{m^{2}}=m^{2}\left[-12 g_{3}^{2} y_{t}^{2}+\frac{9}{4} y_{t}^{4}+36 y_{t}^{2} \lambda-\frac{81}{8} y_{t}^{2} g^{2}-\frac{35}{8} y_{t}^{2} g^{2}\right.
$$$$
\left.+54 \lambda^{2}-27 \lambda g^{2}-9 \lambda g^{2}+\frac{249}{32} g^{4}+\frac{45}{16} g^{2} g^{\prime 2}-\frac{55}{32} g^{\prime 4}\right],
$$

$$
\begin{aligned}
c_{2,1}^{m^{2}}= & m^{2}\left[10 g_{3}^{2} y_{t}^{2}-\frac{27}{8} y_{t}^{4}-18 y_{t}^{2} \lambda+\frac{45}{16} y_{t}^{2} g^{2}+\frac{85}{48} y_{t}^{2} g^{2}\right. \\
& \left.-15 \lambda^{2}+18 \lambda g^{2}+6 \lambda g^{\prime 2}-\frac{145}{64} g^{4}+\frac{15}{32} g^{2} g^{\prime 2}+\frac{557}{192} g^{\prime 4}\right],
\end{aligned}
$$

$$
\begin{gathered}
c_{1,1}^{y_{t}}=y_{t}\left[-4 g_{3}^{2}+\frac{9}{4} y_{t}^{2}-\frac{9}{8} g^{2}-\frac{17}{24} g^{\prime 2}\right], \\
c_{1,1}^{g}=-19 g^{3} / 12, \\
c_{1,1}^{g^{\prime}}=41 g^{\prime 3} / 12 .
\end{gathered}
$$

These counterterm coefficients can be obtained from the 2-loop beta functions and anomalous dimension given in Refs. [20,32-35]; see for example the discussion in

Eqs. (4.5)-(4.14) of Ref. [21] which uses the same notations and conventions as the present paper.

The 1-loop and 2-loop integrals are reduced, using the Tarasov algorithm [36] implemented in the program TARCER [37], to a set of Euclidean $d$-dimensional scalar basis integrals with topologies illustrated in Fig. 1 and defined in our notation in Refs. [38,39]. The 1-loop integrals are

$$
\mathbf{A}(x), \quad \mathbf{B}(x, y),
$$

and the 2-loop integrals are

$$
\begin{gathered}
\mathbf{I}(x, y, z), \quad \mathbf{S}(x, y, z), \quad \mathbf{T}(x, y, z), \\
\mathbf{U}(x, y, z, u), \quad \mathbf{M}(x, y, z, u, v),
\end{gathered}
$$

where the arguments are bare squared masses. The integrals $\mathbf{B}, \mathbf{S}, \mathbf{T}, \mathbf{U}$, and $\mathbf{M}$ also each have an implicit dependence on the external momentum invariant $s=-p^{2}$. The integrals have invariances under interchanges of squared mass arguments that are obvious from the figures.

In terms of bare quantities, the propagators in the selfenergy integrals depend on the squared masses of the neutral and charged Goldstone bosons, the Higgs boson, the top quark, and the $W$ and $Z$ bosons:

$$
\begin{gathered}
G_{B}=m_{B}^{2}+\lambda_{B} v_{B}^{2}, \\
H_{B}=m_{B}^{2}+3 \lambda_{B} v_{B}^{2}, \\
t_{B}=y_{t B}^{2} v_{B}^{2} / 2, \\
W_{B}=g_{B}^{2} v_{B}^{2} / 4, \\
Z_{B}=\left(g_{B}^{2}+g_{B}^{\prime 2}\right) v_{B}^{2} / 4,
\end{gathered}
$$

with a massless photon and ghosts. We then perform an expansion using Eqs. (2.5)-(2.24), to write these quantities in terms of the corresponding $\overline{\mathrm{MS}}$ squared masses.

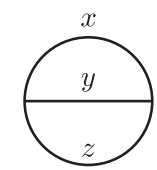

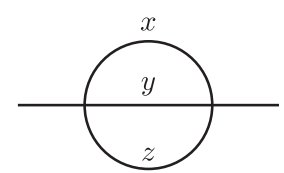

$S$

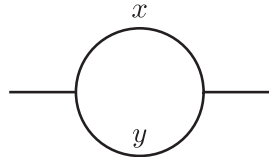

$B$

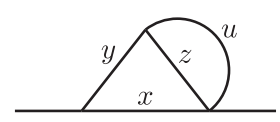

$U$

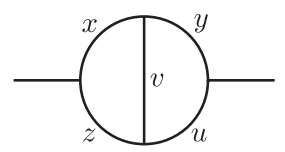

M

FIG. 1. Topologies for the one- and two-loop vacuum and self-energy scalar basis integrals used in this paper and defined in Refs. $[38,39]$. The dot in the $T$ topology stands for a derivative with respect to the squared mass $x$. 
For the 2-loop integrals, this merely requires replacing the bare squared mass arguments by their $\overline{\mathrm{MS}}$ counterparts, because the difference is of 3-loop order. For the 1-loop integrals, the functions $\mathbf{A}$ and $\mathbf{B}$ are expanded to first order around the $\overline{\mathrm{MS}}$ squared-mass arguments

$$
\begin{gathered}
G=m^{2}+\lambda v^{2}, \\
H=m^{2}+3 \lambda v^{2}, \\
t=y_{t}^{2} v^{2} / 2, \\
W=g^{2} v^{2} / 4, \\
Z=\left(g^{2}+g^{\prime 2}\right) v^{2} / 4,
\end{gathered}
$$

using

$$
\begin{aligned}
\mathbf{A}(X)= & \mathbf{A}(x)+(X-x) \frac{\partial}{\partial x} \mathbf{A}(x)+\cdots, \\
\mathbf{B}(X, Y)= & \mathbf{B}(x, y)+(X-x) \frac{\partial}{\partial x} \mathbf{B}(x, y) \\
& +(Y-y) \frac{\partial}{\partial y} \mathbf{B}(x, y)+\cdots,
\end{aligned}
$$

where the derivatives are given in the Appendix. As a further refinement, the parameter $m^{2}$ is eliminated using the minimization condition of the Landau gauge effective potential, which takes the form

$G=m^{2}+\lambda v^{2}=-\frac{1}{16 \pi^{2}} \hat{\Delta}_{1}-\frac{1}{\left(16 \pi^{2}\right)^{2}} \hat{\Delta}_{2}-\cdots$,

given in Eqs. (4.18)-(4.21) of Ref. [22] (with equivalent results in [23]). Here the quantities $\hat{\Delta}_{1}$ and $\hat{\Delta}_{2}$ depend on $t, W, Z$, and

$$
h=2 \lambda v^{2},
$$

but not on $G$ or $H$ or $m^{2}$. The 1-loop integrals involving $G$ as an argument are expanded using Eqs. (A7)-(A14) of the Appendix, while those involving $H$ as an argument are expanded using Eqs. (2.37) and (2.38) again.

The loop integrals are then rewritten in terms of the basis of $\epsilon$-independent integrals

$$
\begin{gathered}
A(x), \quad B(x, y), \quad I(x, y, z), \quad S(x, y, z), \\
T(x, y, z), \quad \bar{T}(0, x, y), U(x, y, z, u), \quad M(x, y, z, u, v) \quad(2.41)
\end{gathered}
$$

which are obtained from the corresponding integrals in Eqs. (2.25) and (2.26) by subtracting appropriate subdivergences and taking the limit $\epsilon \rightarrow 0$. Here $\bar{T}(x, y, z) \equiv$ $T(x, y, z)+B(y, z) \overline{\ln }(x)$ with

$$
\overline{\ln }(x) \equiv \ln \left(x / Q^{2}\right)
$$

The reason for the definition of the function $\bar{T}(x, y, z)$ is that it is well defined as $x \rightarrow 0$, while $T(x, y, z)$ diverges in that limit. For the precise definitions of the integrals in Eq. (2.41), see Sec. 2 of [39]. These integrals also have an implicit dependence on the common external momentum invariant $s$ and on the $\overline{\mathrm{MS}}$ renormalization scale $Q$. In the resulting expression on the right-hand side of Eq. (2.4), there are terms proportional to $s_{\text {pole }} / \epsilon$ and $s_{\text {pole }} / \epsilon^{2}$, corresponding to the Higgs wave function renormalization. These are moved to the left-hand side to allow $s_{\text {pole }}$ to be solved for. Finally, the regulator is removed by taking the limit $\epsilon \rightarrow 0$.

The result for the Higgs squared pole mass is thus obtained in the form:

$$
\begin{aligned}
M_{h}^{2}-i \Gamma_{h} M_{h}= & 2 \lambda v^{2}+\frac{1}{16 \pi^{2}} \Delta_{M_{h}^{2}}^{(1)} \\
& +\frac{1}{\left(16 \pi^{2}\right)^{2}}\left[\Delta_{M_{h}^{2}}^{(2), \mathrm{QCD}}+\Delta_{M_{h}^{2}}^{(2), \text { non-QCD }}\right],
\end{aligned}
$$

where the right-hand side is a function of $v, \lambda, y_{t}, g, g^{\prime}$, $g_{3}, Q$, with propagator masses expressed as the combinations $h, t, W, Z$, and 0 . Working to 2-loop order with bottom, tau, and charm Yukawa couplings neglected, we can treat $s_{\text {pole }}$ as real where it appears as the (implicit) argument of the basis integral functions, and so replace it by $M_{h}^{2}$. This is because the imaginary part of $s_{\text {pole }}$ is already of 2-loop order, and so the effect of including it would make a difference of 3-loop electroweak order in the pole mass. If the lighter fermions are included in the 1-loop self-energy (see below), then there is a 1-loop imaginary part to the complex pole squared mass, but it is numerically smaller than a typical 3-loop order contribution due to the small Yukawa couplings of $b, \tau, c$, so that it can still be safely and consistently neglected. This feature is of course related to the very narrow Higgs width in the standard model. For simplicity, we will therefore write $s=M_{h}^{2}$ below.

The complete lists of 1-loop and 2-loop basis integrals appearing on the right-hand side are

$$
I^{(1)}=\{B(t, t), B(h, h), B(W, W), B(Z, Z), A(t), A(h), A(W), A(Z)\}
$$

and 


$$
\begin{aligned}
I^{(2)}= & \{M(h, h, h, h, h), U(h, h, h, h), S(h, h, h), M(h, Z, h, Z, Z), U(h, h, Z, Z), \\
& M(W, W, W, W, h), U(W, W, W, h), S(h, W, W), T(W, W, h), \\
& M(Z, Z, Z, Z, h), U(Z, Z, Z, h), S(h, Z, Z), T(Z, Z, h), \\
& M(W, W, W, W, Z), U(W, W, W, Z), S(W, W, Z), T(W, W, Z), T(Z, W, W), \\
& M(W, Z, W, Z, W), U(Z, Z, W, W), M(h, W, h, W, W), U(h, h, W, W), \\
& M(t, t, t, t, Z), U(t, t, t, Z), S(t, t, Z), T(t, t, Z), T(Z, t, t), \\
& M(t, t, t, t, h), U(t, t, t, h), S(h, t, t), T(t, t, h), \\
& M(t, Z, t, Z, t), U(Z, Z, t, t), M(t, h, t, h, t), U(h, h, t, t), \\
& M(t, W, t, W, 0), U(W, W, 0, t), U(t, t, 0, W), S(0, t, W), T(W, 0, t), T(t, 0, W), \\
& M(t, t, t, t, 0), T(t, 0, t), \bar{T}(0, t, t), \\
& M(W, W, W, W, 0), T(W, 0, W), \bar{T}(0, W, W), U(W, W, 0,0), S(0,0, W), \\
& T(W, 0,0), U(Z, Z, 0,0), S(0,0, Z), T(Z, 0,0), I(h, h, h), I(t, t, Z), \\
& I(h, t, t), I(W, W, Z), I(h, W, W), I(h, Z, Z), I(0, t, W), I(0, h, W), \\
& I(0, h, Z), I(0, W, Z), I(0,0, W), I(0,0, Z), I(0,0, h), I(0,0, t)\} .
\end{aligned}
$$

In each of the $B, S, T, \bar{T}, U$, and $M$ integrals, the external momentum invariant is taken to be the real pole squared mass, $s=M_{h}^{2}$, as discussed above. Then Eq. (2.43) can be solved numerically, by iteration.

The explicit results for the 1-loop part and the 2-loop QCD part of the Higgs pole squared mass corrections are

$$
\begin{aligned}
\Delta_{M_{h}^{2}}^{(1)}= & 3 y_{t}^{2}(4 t-s) B(t, t)-18 \lambda^{2} v^{2} B(h, h) \\
+ & \frac{1}{2}\left(g^{2}+g^{\prime 2}\right)\left[\left(s-3 Z-s^{2} / 4 Z\right) B(Z, Z)\right. \\
- & s A(Z) / 2 Z+2 Z] \\
+ & g^{2}\left[\left(s-3 W-s^{2} / 4 W\right) B(W, W)\right. \\
- & s A(W) / 2 W+2 W], \\
\Delta_{M_{h}^{2}}^{(2), \mathrm{QCD}}= & g_{3}^{2} y_{t}^{2}[8(4 t-s)(s-2 t) M(t, t, t, t, 0) \\
& +(36 s-168 t) T(t, 0, t)+16(s-4 t) \bar{T}(0, t, t) \\
& +14 s B(t, t)^{2}+(-176+36 s / t) A(t) B(t, t) \\
& \left.+(80 t-36 s) B(t, t)-28 A(t)^{2} / t+80 t-17 s\right] .
\end{aligned}
$$

In Eq. (2.46), a term $3 \lambda\left(s^{2}-h^{2}\right) B(0,0) / h$ coming from loops involving Goldstone bosons and the unphysical modes of the vector bosons has been moved into the 2-loop order non-QCD part discussed below, by iterating using $s=h+\Delta_{M_{h}^{2}}^{(1)} / 16 \pi^{2}$. There, it cancels against other terms, and the full 2-loop result does not depend on $B(0,0)$. This is as expected, because a term with $B(0,0)$ coming from loops involving Goldstone bosons and the unphysical modes of the vector bosons would imply an imaginary part to the pole squared mass that does not correspond to any physical decay of the Higgs boson. One-loop contributions $B(0, Z)$ and $B(0, W)$ from individual Feynman diagrams involving Goldstone bosons and the unphysical modes of the vector bosons also cancel as expected, even without iteration in $s$.

For the remaining, non-QCD, 2-loop contributions, there are a large number of terms, and some of them are a bit complicated, so that the length of the result may exceed the threshold of impoliteness, and we decline to present them explicitly in print. The result has the form:

$$
\begin{aligned}
\Delta_{M_{h}^{2}}^{(2) \text { non-QCD }}= & \sum_{i} c_{i}^{(2)} I_{i}^{(2)}+\sum_{j \leq k} c_{j, k}^{(1,1)} I_{j}^{(1)} I_{k}^{(1)} \\
& +\sum_{j} c_{j}^{(1)} I_{j}^{(1)}+c^{(0)} .
\end{aligned}
$$

The coefficients $c_{i}^{(2)}$ and $c_{j, k}^{(1,1)}$ and $c_{j}^{(1)}$ and $c^{(0)}$ are available in electronic form in a file called COEFFICIENTS.TXT. They are also implemented in a public computer code written in $\mathrm{C}$, described below. These electronic files are available from the authors' web pages [40], and COEFFICIENTS.TXT is also included as an ancillary file with the arXiv source for this article. In these coefficients, we replaced $s$ by its tree-level approximation $2 \lambda v^{2}$ wherever it appears explicitly (but not where it appears as the implicit argument of the basis functions). This enforces the cancellations between Goldstone and unphysical vector boson contributions, avoiding spurious imaginary contributions to the pole squared mass that do not correspond to physical decay modes of the Higgs boson. Therefore each coefficient is a sum of ratios of polynomials in $\lambda, y_{t}, g, g^{\prime}$, 
multiplied by the appropriate power of $v$. The impact incurred by doing these substitutions for $s$ is of 3-loop order without involving QCD, and so is beyond the order of our calculations in this paper, including the QCD part of the leading 3-loop corrections discussed in the next section.

The expression of the result in terms of the basis integrals is not unique, because there are identities between different basis integrals that hold when the squared mass arguments are not generic. These identities include Eqs. (A.14), (A.15), and (A.17)-(A.20) in Ref. [41], and Eqs. (A17)-(A21) in the Appendix of the present paper. We also used the threshold integral relations (A15) and (A16) in the Appendix to simplify the 2-loop order non-QCD part.

There are several quite nontrivial checks on the calculation. First, we checked that all single and double poles in $\epsilon$ cancel in $M_{h}^{2}$. This relies on agreement between the counterterm poles $c_{\ell, n}^{X}$ (for $X=v, \lambda, m^{2}, y_{t}, g, g^{\prime}$ ) as extracted from the Higgs anomalous dimension and the beta functions in the literature, and the divergent parts of the loop integrations performed independently here. Second, we checked that logarithms of $G$ cancel, avoiding any spurious imaginary parts that would occur if the renormalization scale were chosen so that $G<0$, or spurious divergences that would occur if $G=0$. Third, we observed cancellation between the parts of loop integral functions involving Landau gauge vector propagators with poles at squared mass equal to 0 and the corresponding Goldstone propagators, once the latter were expanded using Eq. (2.39). This is important in verifying the absence of spurious absorptive (imaginary) parts of the self-energy evaluated on shell. Fourth, we noted that the imaginary part $-i \Gamma_{h} M_{h}$ of Eq. (2.43) comes entirely from the contributions of the six basis integrals $U(W, W, 0,0), S(0,0, W), T(W, 0,0)$ and $U(Z, Z, 0,0), S(0,0, Z), T(Z, 0,0)$, corresponding to the 3-body decays $\Gamma\left(h \rightarrow W f^{\prime}\right)$ and $\Gamma(h \rightarrow Z f \bar{f})$. We checked numerically to very high precision that these imaginary contributions, when computed with $s=h$, agree with the tree-level prediction for the 3-body widths found in Eqs. (8a)-(10) of Ref. [42]. Fifth, we checked that although some of the individual 2-loop coefficients in Eq. (2.48) are singular in the formal limits $g, g^{\prime} \rightarrow 0$ or $\lambda \rightarrow 0$, the whole expression is well behaved in those limits, thanks to relations between different basis integrals when squared mass arguments are small. Finally, we checked that the result for $M_{h}^{2}$ is renormalization group scale invariant through terms of 2-loop order. This is in principle equivalent to the first check mentioned, but in practice it tests the validity of various intermediate steps. It takes the form:

$0=Q \frac{d}{d Q} M_{h}^{2}=\left[Q \frac{\partial}{\partial Q}-\gamma_{\phi} v \frac{\partial}{\partial v}+\sum_{X} \beta_{X} \frac{\partial}{\partial X}\right] M_{h}^{2}$,

where $X=\left\{\lambda, y_{t}, g, g^{\prime}, g_{3}\right\}$, and $\gamma_{\phi}$ is the anomalous dimension of the Higgs field. This check makes use of the derivatives of basis integrals with respect to the implicit argument $Q$, provided in Eqs. (4.7)-(4.13) of Ref. [38], and on Eqs. (A5) and (A6) in the Appendix of the present paper. It also makes use of the $\overline{\mathrm{MS}}$ beta functions and Higgs anomalous dimension given in Refs. [20,32-35,43,44].

Although the lighter quarks and leptons have been neglected above due to their very small Yukawa couplings, it is easy enough to include them in the leading approximation:

$\Delta_{M_{h}^{2}}^{(1), b, \tau, c, \ldots}=-\left[3 y_{b}^{2}+y_{\tau}^{2}+3 y_{c}^{2}+\cdots\right] B(0,0) M_{h}^{2}$.

Here we have taken $s=M_{h}^{2}$ and dropped the $y_{f}^{4}$ contributions and replaced the masses in light fermion propagators by 0 . In that limit, we can also take

$$
B(0,0)=2-\ln \left(M_{h}^{2} / Q^{2}\right)+i \pi .
$$

The numerical impact on the real pole mass $M_{h}$ from Eq. (2.50) is seen to be of order $1 \mathrm{MeV}$. By comparing the imaginary part of the pole squared mass, $M_{h}^{2}-i \Gamma_{h} M_{h}$, to the contribution of Eq. (2.50), multiplied by the loop factor $1 / 16 \pi^{2}$, we also obtain the well-known result

$$
\Gamma(h \rightarrow f \bar{f})=\frac{N_{c} y_{f}^{2}}{16 \pi} M_{h} .
$$

However, there are certainly better ways of obtaining the precise Higgs decay widths in the standard model; see for example Ref. [45] and references therein.

\section{LEADING THREE-LOOP CORRECTIONS TO THE HIGGS MASS}

In this section, we find the leading 3-loop contributions to the Higgs pole squared mass in the effective potential approximation, based on the formal limit in which the top-quark squared mass is taken to be much larger than the squared masses of $h, Z$, and $W$. In that limit, the Higgs selfenergy function at leading order in $y_{t}$ and $g_{3}$ can be approximated by taking $s=0$, and is proportional to the second derivative of the renormalized effective potential with respect to the Higgs field. Taking into account also the change in the minimization condition of the effective potential, we have a contribution (see for example Sec. VI of Ref. [21]):

$$
\delta M_{h}^{2}=\left[\frac{\partial^{2}}{\partial v^{2}}-\frac{1}{v} \frac{\partial}{\partial v}\right] \delta V_{\text {eff }} .
$$

Using the leading 3-loop effective potential of Ref. [21], with resummed Goldstone boson contributions to eliminate spurious imaginary parts and infrared singular contributions [22,23], we obtain the 3-loop contribution to be added to Eq. (2.43):

$\Delta M_{h}^{2}=\frac{1}{\left(16 \pi^{2}\right)^{3}}\left[\Delta_{M_{h}^{2}}^{(3), \text { leading } \mathrm{QCD}}+\Delta_{M_{h}^{2}}^{(3) \text { leading non-QCD }}\right]$ 
where

$$
\begin{aligned}
\Delta_{M_{h}^{2}}^{(3) \text { leading QCD }}= & g_{3}^{4} y_{t}^{2} t[248.122+839.197 \overline{\ln }(t) \\
& \left.+160 \overline{\ln }^{2}(t)-736 \overline{\ln }^{3}(t)\right] \\
& +g_{3}^{2} y_{t}^{4} t[2764.365+1283.716 \overline{\ln }(t) \\
& \left.-360 \overline{\ln }^{2}(t)+240 \overline{\ln }^{3}(t)\right],
\end{aligned}
$$

$$
\begin{aligned}
\Delta_{M_{h}^{2}}^{(3) \text { leading non-QCD }}= & y_{t}^{6} t[-3199.017+36 \overline{\ln }(h) \\
& -2653.511 \overline{\ln }(t)+756 \overline{\ln }(h) \overline{\ln }(t) \\
& \left.+\frac{27}{2} \overline{\ln }^{2}(t)+324 \overline{\ln }(h) \overline{\ln }^{2}(t)-225 \overline{\ln }^{3}(t)\right] .
\end{aligned}
$$

The analytical forms of the decimal coefficients are

$$
\begin{aligned}
& 248.122 \approx- \frac{3776}{9}+320 \zeta(3)+\frac{704 \pi^{4}}{135} \\
&+ \frac{256}{9} \ln ^{2}(2)\left[\pi^{2}-\ln ^{2}(2)\right]-\frac{2048}{3} \mathrm{Li}_{4}(1 / 2), \\
& 839.197 \approx 128 \zeta(3)+2056 / 3
\end{aligned}
$$

$$
\begin{aligned}
2764.365 \approx & \frac{760}{3}-\frac{16 \pi^{2}}{3}+576 \zeta(3)+\frac{496 \pi^{4}}{15} \\
& +\frac{512}{3} \ln ^{2}(2)\left[\pi^{2}-\ln ^{2}(2)\right]-4096 \mathrm{Li}_{4}(1 / 2),
\end{aligned}
$$

$$
\begin{gathered}
1283.716 \approx-344+48 \pi^{2}+960 \zeta(3), \\
-3199.017 \approx-727-\frac{17 \pi^{2}}{2}-1962 \zeta(3)-\frac{88 \pi^{4}}{15} \\
+32 \ln ^{2}(2)\left[\pi^{2}-\ln ^{2}(2)\right]+768 \mathrm{Li}_{4}(1 / 2), \\
-2653.511 \approx-\frac{4191}{2}-39 \pi^{2}-144 \zeta(3) .
\end{gathered}
$$

The 3-loop approximate formulas just described may be subject to significant corrections, because $s / t \approx 0.59$ is not a very small expansion parameter. However, experience shows that in such small-s expansions of loop integrals the coefficients of $s / t$ are typically also less than 1 , so that the 3-loop approximation above might be expected to provide the bulk of the effect. For example, the small $s$-expansions of the 1-loop and 2-loop basis functions involved in the contributions from the top quark and gluons are [38]

$$
B(t, t)=-\overline{\ln }(t)+\frac{s}{6 t}+\cdots
$$

$$
\begin{gathered}
T(t, 0, t)=\frac{1}{2}[\overline{\ln }(t)-1]^{2}+\frac{s}{4 t}+\cdots \\
\bar{T}(0, t, t)=\frac{1}{2}\left[3+2 \overline{\ln }(t)+\overline{\ln }^{2}(t)\right]+\frac{s}{36 t}[6 \overline{\ln }(t)+1]+\cdots \\
M(t, t, t, t, 0)=\frac{1}{t}+\frac{13 s}{72 t^{2}}+\cdots
\end{gathered}
$$

As noted in the discussion surrounding Eqs. (6.21)-(6.28) of Ref. [21], the relatively small coefficient 248.122 of the $g_{3}^{4} y_{t}^{2} t$ term independent of $\ln (t)$ in Eq. (3.3) of the present paper is the result of a remarkable accidental near-cancellation. Because of this, the $g_{3}^{2} y_{t}^{4} t$ and $y_{t}^{6} t$ contributions are actually numerically more important than the $g_{3}^{4} y_{t}^{2} t$ contribution.

Because the full $s$ dependence of the 2-loop QCD part was retained above, the QCD part of the 3-loop contribution found in the effective potential approximation can simply be added in. As a check, we have verified the renormalization group invariance of the combined full 2-loop plus leading 3-loop QCD result from Eqs. (2.43) and (2.46)-(2.48) and Eqs. (3.2)-(3.3). This check consists of evaluating Eq. (2.49) including all terms of 2-loop order and the terms of 3-loop order that involve $g_{3}$ and are not suppressed by $\lambda, g$, or $g^{\prime}$. The check again makes use of the $\overline{\mathrm{MS}}$ beta functions and Higgs anomalous dimension given in Refs. [20,32-35,43,44], as well as Eqs. (4.7)-(4.13) of Ref. [38], and on Eqs. (A5) and (A6) in the Appendix of the present paper.

For the 3-loop non-QCD part, the situation is more subtle, because in the 2-loop non-QCD contribution of Eq. (2.48) we made the substitution $s=h$, implicitly dropping 3-loop order corrections of order $y_{t}^{6} t$, formally of the same order as in Eq. (3.4). However, the approximation for the 3-loop contribution above is still justified if the renormalization scale $Q$ is chosen within an appropriate range. To see this, note that if $Q$ is chosen to the particular value such that $s=h$, then the numerical error made by using $s=h$ in the 2-loop part will vanish exactly. More formally, since we are interested in the 3-loop contributions in the limits $s / t \ll 1$ and $y_{t} \gg \lambda, g, g^{\prime}$, note that from Eqs. (2.43) and (2.46) we have

$$
s=h-\frac{1}{16 \pi^{2}} 12 y_{t}^{2} t \overline{\ln }(t)+\cdots
$$

where the ellipses represent electroweak terms and terms suppressed by $s / t$. Thus we see that the neglected 3-loop order terms that are of order $y_{t}^{6} t$ will vanish when $Q$ is chosen so that $\overline{\ln }(t)=0$, and are correspondingly suppressed for small $\overline{\ln }(t)$. In practice, the conditions $s=h$ and $\overline{\ln }(t)=0$ imply values of $Q$ that are not very far apart from each other, and therefore this range of $Q$ is preferred when including the 3-loop contributions above. As we will 
see below, the numerical renormalization scale dependence of the computed $M_{h}$ is mild for a larger range of $Q$.

\section{COMPUTER CODE IMPLEMENTATION AND NUMERICAL RESULTS}

We have implemented the Higgs pole mass calculations described above in a computer code library of utilities written in C, called SMH (for "standard model Higgs"). The code can be downloaded from the authors' web pages [40].

The SMH program requires the use of the program TSIL (two-loop self-energy integral library) [39], which is used to handle the loop integrations. The 1-loop basis integrals are evaluated in terms of logarithms, and the last 29 of the 2-loop integrals in the list Eq. (2.45) [starting with $S(0, t, W)]$ are computed analytically in terms of polylogarithms by TSIL, using formulas obtained in [6,38,46-51]. The other 38 integrals are computed numerically by TSIL; this requires only 12 calls of the function TSIL_EvALUATE. The program SMH is distributed with a file README.TXT, which gives complete instructions for building and using it, as well as several example and test programs. Most user applications, like the example programs provided, will make use of a static archive called LIBSMH.A, which can be linked to by $\mathrm{C}$ or $\mathrm{C}++$ programs.

The functionality implemented in SMH includes the following:

(i) $\mathrm{SMH}$ _RGRUN performs the renormalization group running of $\lambda, y_{t}, g_{3}, g, g^{\prime}, m^{2}, v$ at up to 3-loop order, using the $\overline{\mathrm{MS}}$ beta functions and Higgs anomalous dimension given in Refs. [20,32-35,43,44]. (At this writing, the lighter fermion Yukawa couplings $y_{b}, y_{\tau}, y_{c}$ are not included, but they will be in a future release, as an option.)

(ii) SMH_FIND_VEV and SMH_FIND_M2 implement the minimization of the Landau gauge effective potential for the standard model, at up to 2-loop order [20] with leading 3-loop corrections [21], using Eqs. (4.18)-(4.21) of Ref. [22]. The function SMH_FIND_VEV finds $v$, given $m^{2}, \lambda, y_{t}, g, g^{\prime}, g_{3}$ at a renormalization scale $Q$, while the function SMH_FIND_M2 does the inverse task of finding $\mathrm{m}^{2}$, given $v, \lambda, y_{t}, g, g^{\prime}, g_{3}$ at $Q$.

(iii) SMH_FIND_MH and SMH_FIND_LAMBDA implement the 2-loop Higgs pole mass of Eqs. (2.43) and (2.46)-(2.48), with the leading 3-loop corrections from Eqs. (3.2)-(3.4). The function SMH_FIND_MH finds $M_{h}$ given $\lambda, v, y_{t}, g, g^{\prime}, g_{3}$ at $Q$, while the function SMH_FIND_LAMBDA does the inverse, finding $\lambda$ given $M_{h}$ and $v, y_{t}, g, g^{\prime}, g_{3}$ at $Q$.

The user can choose various different loop-order approximations, as illustrated in the examples below, with the default being to use the complete set of available corrections. Stand-alone command-line programs corresponding to each of the above library functions are also included in the SMH package. We also include example programs that produce the data for the figures below. We plan to maintain and improve the SMH code indefinitely, and welcome bug reports or suggestions.

For purposes of illustration, consider as benchmark inputs [taken from Ref. [19] version 2, Eqs. (55)-(59)]:

$$
\begin{gathered}
m^{2}\left(M_{t}\right)=-(93.36 \mathrm{GeV})^{2}, \\
\lambda\left(M_{t}\right)=0.12711, \\
y_{t}\left(M_{t}\right)=0.93558, \\
g_{3}\left(M_{t}\right)=1.1666, \\
g\left(M_{t}\right)=0.64822, \\
g^{\prime}\left(M_{t}\right)=0.35761,
\end{gathered}
$$

where $Q=M_{t}=173.10 \mathrm{GeV}$ is the input scale. From these, we find our benchmark value by minimizing the effective potential with leading 3-loop corrections:

$$
v\left(M_{t}\right)=247.039 \mathrm{GeV} .
$$

If only the full 2-loop corrections were included, the result would be $v\left(M_{t}\right)=247.381 \mathrm{GeV}$.

The variation of $v(Q)$ with $Q$ is shown in Fig. 2. To make the figure, the input parameters $m^{2}, \lambda, y_{t}, g_{3}, g, g^{\prime}$ were run from the input scale to $Q$ using 3-loop renormalization group equations. In the left panel of Fig. 2, we show the results for the 2-loop minimization condition of Eqs. (4.18)-(4.20) of Ref. [22] as the dashed line, while the solid line is the 2-loop plus leading 3-loop result obtained by including also Eq. (4.21) of the same reference. The right panel shows the ratio of $v(Q)$ to the value $v_{\text {run }}(Q)$ obtained from directly running it using its renormalization group equation and input value Eq. (4.7). The deviation of this ratio from unity is due to higher order-effects; it is seen to be less than $0.1 \%$ for the calculation that includes the leading 3-loop effects.

In Fig. 3, we reverse the roles of $m^{2}$ and $v$, by showing the dependence of the Higgs Lagrangian mass parameter $m^{2}(Q)$ obtained by minimizing the effective potential, this time with the VEV $v(Q)$ as an input parameter. To make the figure, the input parameters $v, \lambda, y_{t}, g_{3}, g, g^{\prime}$ were run from the input scale to $Q$ using 3-loop renormalization group equations. In the left panel of Fig. 3, we show $\sqrt{-m^{2}}$ obtained from the 2-loop minimization condition of Eqs. (4.18)-(4.20) of Ref. [22] as the dashed line, while the solid line is the 2-loop plus leading 3-loop result obtained by including also Eq. (4.21) of the same reference. The right panel shows the ratio of $m^{2}(Q)$ to the value $m_{\text {run }}^{2}(Q)$ obtained from directly running it using its renormalization group equation and input value Eq. (4.1). 

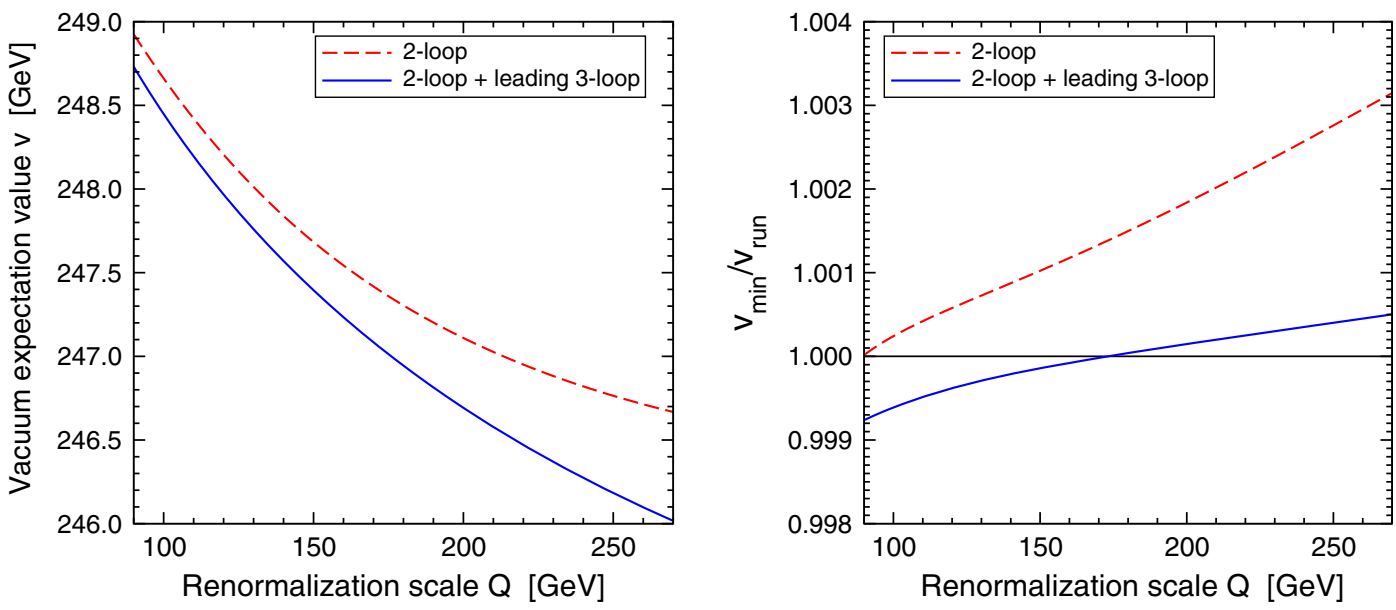

FIG. 2 (color online). The standard model Higgs VEV, $v(Q)$, obtained from minimization of the effective potential is shown in the left panel as a function of the renormalization scale $Q$. The dashed line shows the results for the 2-loop minimization condition of Eqs. (4.18)-(4.20) of Ref. [22], while the solid line is the 2-loop plus leading 3-loop result obtained by including also Eq. (4.21) of the same reference. The input parameters $m^{2}, \lambda, y_{t}, g_{3}, g, g^{\prime}$ are obtained at the scale $Q$ by 3-loop renormalization group running starting from Eqs. (4.1)-(4.6). The right panel shows the ratio of $v(Q)$ to the value $v_{\text {run }}(Q)$ obtained from directly running it using its renormalization group equation and input value Eq. (4.7).

In Fig. 4, we show results for the Higgs pole mass $M_{h}$ as a function of the renormalization scale $Q$. To make the figure, the benchmark input parameters $\lambda, y_{t}, g, g^{\prime}, g_{3}, v$ were run from the input scale to $Q$ using 3-loop renormalization group equations. The lower solid (blue) line is the 2-loop calculation of Eqs. (2.43) and (2.46)-(2.48), while the upper solid (black) line includes also the leading 3-loop contributions of Eqs. (3.2)-(3.4). The results at the input scale $Q=173.1 \mathrm{GeV}$ are $M_{h}=125.789 \mathrm{GeV}$ and $M_{h}=125.818$, respectively. We also show the tree-level approximation $\sqrt{2 \lambda} v$ as the dotted line, and the 1-loop approximation obtained from Eqs. (2.43) and (2.46) as the

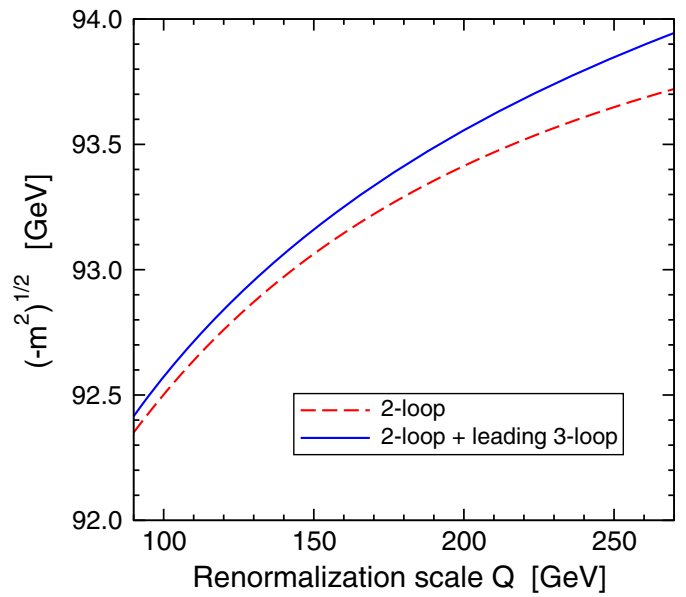

short-dashed line, and the 1-loop approximation with the 2-loop QCD corrections from (2.47) included as the long-dashed line.

Figure 5 is a close-up of the previous figure, to illustrate the scale dependence more clearly for the full 2-loop and leading 3-loop approximations. The lower (blue) line is again the full 2-loop $M_{h}$ as calculated from Eqs. (2.43) and (2.46)-(2.48). For comparison, we also show the result for the full 2-loop plus the 3-loop QCD contribution of Eqs. (3.2)-(3.3), without including the non-QCD 3-loop corrections, as the upper (magenta) line. This has a much stronger scale dependence than the 2-loop result, despite

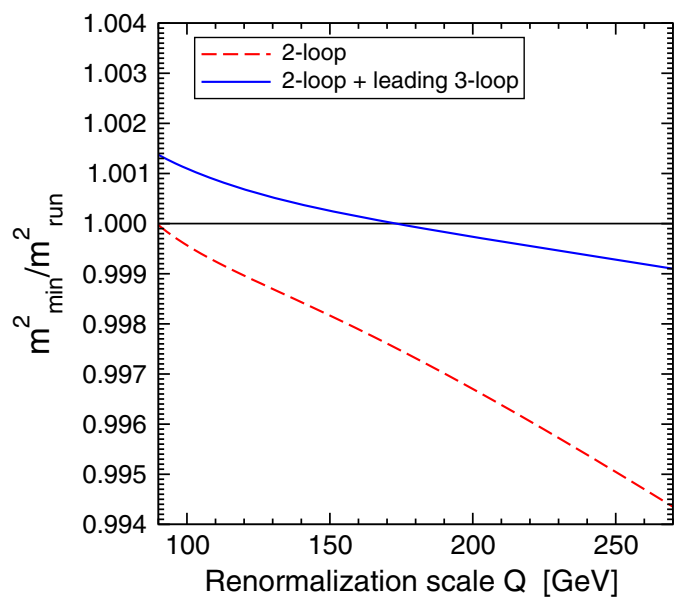

FIG. 3 (color online). The standard model Lagrangian Higgs squared mass parameter, obtained from minimization of the effective potential is shown in the left panel as $\sqrt{-m^{2}}$ as a function of the renormalization scale $Q$. The dashed line shows the results for the 2 loop minimization condition of Eqs. (4.18)-(4.20) of Ref. [22], while the solid line is the 2-loop plus leading 3-loop result obtained by including also Eq. (4.21) of the same reference. The input parameters $v, \lambda, y_{t}, g_{3}, g, g^{\prime}$ are obtained at the scale $Q$ by 3 -loop renormalization group running starting from Eqs. (4.2)-(4.7). The right panel shows the ratio of $m^{2}(Q)$ to the value $m_{\text {run }}^{2}(Q)$ obtained from directly running it using its renormalization group equation and input value Eq. (4.1). 


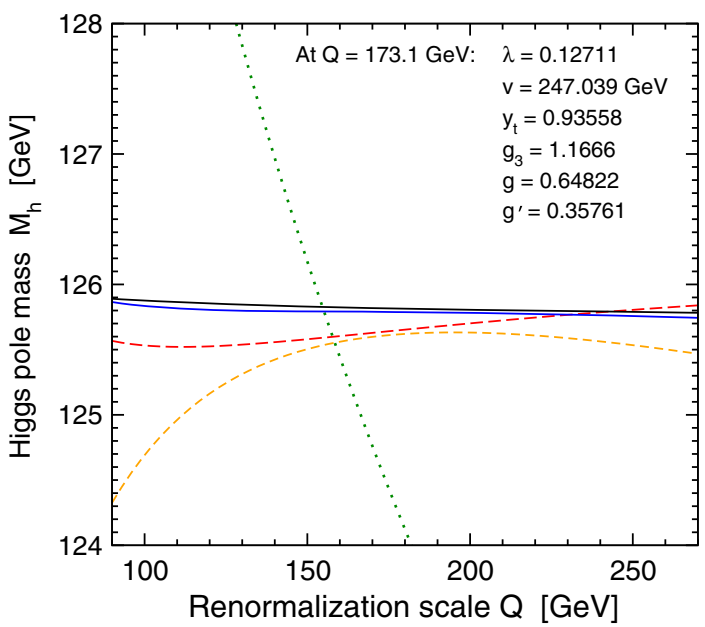

FIG. 4 (color online). The calculated Higgs pole mass $M_{h}$ as a function of the renormalization scale $Q$, in various approximations. The input data at $Q$ are obtained from 3-loop renormalization group running of $\lambda, y_{t}, g, g^{\prime}, g_{3}, v$ starting from Eqs. (4.2)-(4.7). The dotted (green) line is the tree-level approximation $\sqrt{2 \lambda} v$. The short-dashed (orange) line is the 1-loop approximation obtained from Eqs. (2.43) and (2.46). The longdashed (red) line is the 1-loop approximation with the 2-loop QCD corrections from Eq. (2.47). The lower solid (blue) line is the 2-loop $M_{h}$ as calculated from Eqs. (2.43) and (2.46)-(2.48), while the upper solid (black) line also includes the leading 3-loop corrections of Eqs. (3.2)-(3.4).

the formal independence of $M_{h}$ with respect to $Q$ through terms of 3-loop order involving $g_{3}$. Including the non-QCD $y_{t}^{6} t$ contributions from Eq. (3.4) yields the middle (black) line, which again has a mild scale dependence comparable to the 2-loop result. The residual scale dependence is due to higher order effects. Note that, as can be seen by comparing Eqs. (3.3) and (3.4), the 3-loop QCD and 3-loop $y_{t}^{6} t$

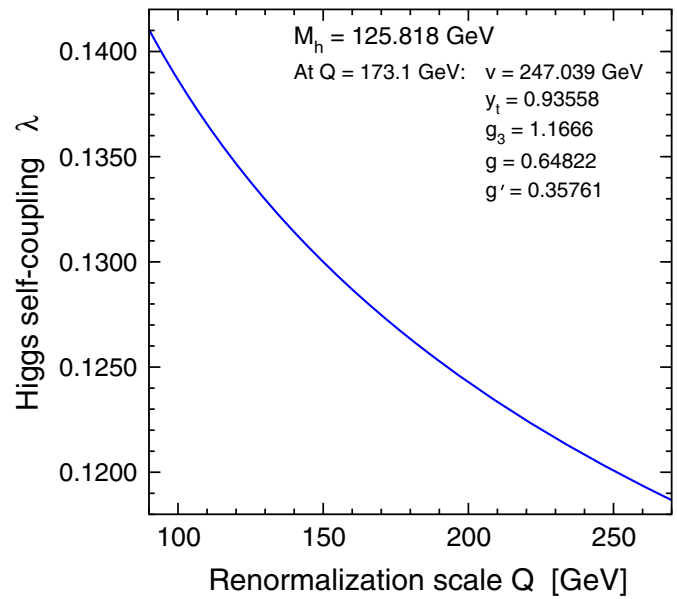

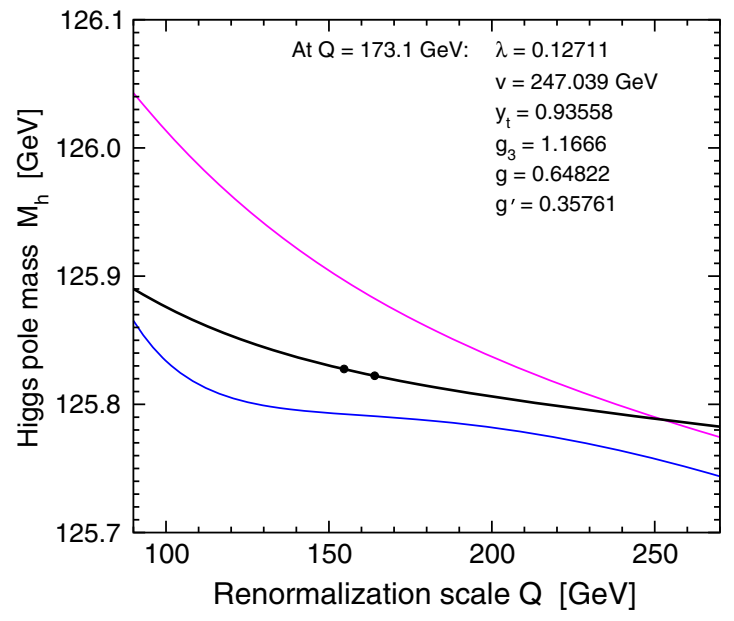

FIG. 5 (color online). A close-up of the dependence of the calculated $M_{h}$ on $Q$, as in Fig. 4. The lower (blue) line is the full 2-loop $M_{h}$ as calculated from Eqs. (2.43) and (2.46)-(2.48). The upper (magenta) line is the full 2-loop plus the 3-loop QCD contribution of Eqs. (3.2)-(3.3), not shown in Fig. 4. The middle (black) line is the full 2-loop plus the 3-loop corrections of Eqs. (3.2)-(3.4), with the left dot marking the case $s=h$ and the right dot marking the case $\overline{\ln }(t)=0$.

contributions contribute with opposite sign, and have an opposite scale dependence.

The points with $s=h$ and $\overline{\ln }(t)=0$ are marked with dots on the leading 3-loop $M_{h}$ line in Fig. 5. As argued in the previous section, the range of $Q$ near these points is preferred due to the treatment of the 2-loop corrections. In particular, the choice of $Q$ that makes $\overline{\ln }(t)=0$ is easy to implement as a natural standard. Given the value of the running top-quark mass, and the observed mild scale dependence in this region, a fixed value of, say, $Q=$ $160 \mathrm{GeV}$ would also make sense.

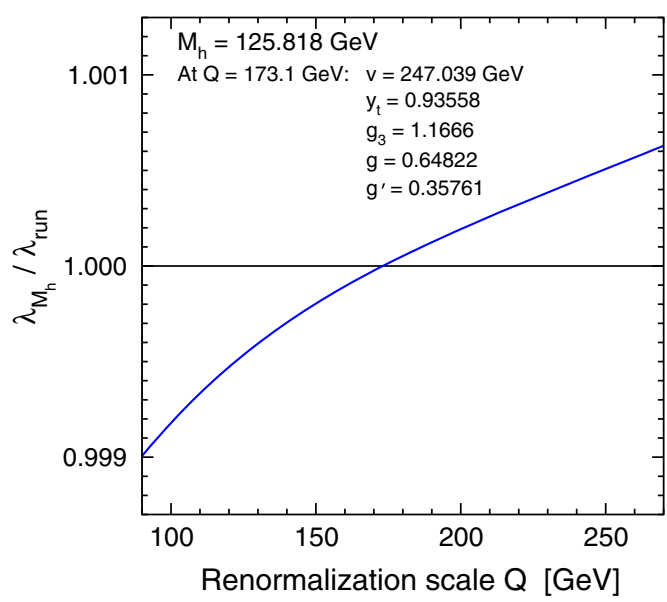

FIG. 6 (color online). The Higgs self-coupling parameter, $\lambda_{M_{h}}(Q)$ as calculated from a fixed pole mass $M_{h}=125.818 \mathrm{GeV}$ using Eqs. (2.43) and (2.46)-(2.48) and Eqs. (3.2)-(3.4), with $y_{t}, g, g^{\prime}, g_{3}, v$ obtained at the scale $Q$ by 3-loop renormalization group running starting from Eqs. (4.3)-(4.7). The right panel shows the ratio of $\lambda_{M_{h}}(Q)$ to the value $\lambda_{\text {run }}(Q)$ obtained from directly running it using its renormalization group equation and input value Eq. (4.2). 
In the left panel of Fig. 6, we show the scale dependence of $\lambda(Q)$ obtained from Eqs. (2.43) and (2.46)-(2.48) and Eqs. (3.2)-(3.4), with the same input parameters $v, y_{t}, g, g^{\prime}, g_{3}$ at $Q=173.1 \mathrm{GeV}$, but now using a fixed pole mass $M_{h}=125.818 \mathrm{GeV}$ as the input. This value is chosen so that the calculated Higgs self-coupling at the input scale agrees with Eq. (4.2). In the right panel, we show the ratio of $\lambda_{M_{h}}(Q)$ determined in this way to $\lambda_{\text {run }}(Q)$ obtained by directly running it from the input value Eq. (4.2) using its 3-loop renormalization group equation. As expected, the ratio is very close to 1 for all values of $Q$; the two versions of $\lambda$ would be visually indistinguishable in the left panel. These results illustrate the renormalization group scale independence through 2-loop and 3-loop QCD order that we verified analytically as described above, with small discrepancies less than $0.1 \%$ coming from 3-loop $y_{t}^{6} t$ and from subleading 3-loop and higherorder effects.

\section{OUTLOOK}

In this paper, we have obtained the pole mass of the Higgs boson, $M_{h}$, including full 2-loop and leading 3-loop corrections, in the $\overline{\mathrm{MS}}$ scheme. The calculation was done in Landau gauge, in order to match with existing multiloop calculations of the effective potential used to eliminate $m^{2}$ by relating it to the VEV $v$ (defined in this paper as the minimum of the full effective potential) and the other Lagrangian parameters. The inputs to the calculation are the $\overline{\mathrm{MS}}$ running parameters of the theory, $v, \lambda, y_{t}, g, g^{\prime}, g_{3}$. Other observables, such as the pole masses of the top quark and the $W, Z$ bosons, are not inputs to the calculation, and are to be calculated separately. A possible advantage to this strategy is that future refinements in calculations and measurements of those other observable quantities will not be entangled with the calculation of the Higgs pole mass. Previous results for the 2-loop corrections [17-19] to the Higgs mass were organized in a different way, and in the case of the non-QCD corrections [18,19] were given only in the form of simple interpolating formulas, making comparison with the present paper not practical. Our full analytic results are contained in an ancillary electronic file, and a computer code called SMH is provided [40], implementing the results for $M_{h}$, the effective potential minimization, and renormalization group running.

Because there is no way of directly measuring the Higgs self-coupling parameter accurately in the immediate future, the measurement of the Higgs mass is the best way to determine $\lambda$, assuming the validity of the standard model, with variations related approximately by

$$
\Delta \lambda=0.00205\left(\Delta M_{h} / \mathrm{GeV}\right)
$$

From the renormalization scale variation and the magnitudes of the leading 3-loop QCD and non-QCD effects, we make a very rough estimate of the theoretical uncertainty on $M_{h}$ of $100 \mathrm{MeV}$, or about $0.1 \%$, taking $\overline{\mathrm{MS}}$ quantities as the inputs. This does not include the effects of reducible parametric error, notably the dependence on the uncertainties in the top-quark Yukawa coupling (or mass) and the QCD coupling. The future experimental error in $M_{h}$ has been estimated [52] to be perhaps $100 \mathrm{MeV}$ (50 MeV) with $300 \mathrm{fb}^{-1}$ (respectively $3000 \mathrm{fb}^{-1}$ ) at the LHC, and of order $30 \mathrm{MeV}$ or less at future $e^{+} e^{-}$colliders. We conclude that more refined 3-loop order and quite possibly 4-loop order corrections to $M_{h}$ will be necessary in order to make the theoretical error small compared to the foreseeable experimental error, discounting the parametric uncertainties that may be reducible by independent calculations and measurements. At the least, a further refinement of the 3-loop $M_{h}$ calculation would serve to firm up an estimate of the theoretical error.

Besides applications within the standard model, the result may find use in extensions of the standard model, including supersymmetry. The most straightforward interpretation of the current LHC searches for supersymmetry is that the superpartners, if they exist, are sufficiently heavy that the standard model can be treated as an effective theory with other new physics nearly decoupled. The direct observation that the Higgs mass is relatively large compared to most pre-LHC expectations within supersymmetry can be taken as indirect evidence of the same thing. In the past, many attempts to compute the Higgs mass within supersymmetry have calculated directly within the full softly broken supersymmetric theory in the Feynman diagrammatic [53-61] and effective potential approximation [62-65] approaches. However, it now seems to us that with very heavy superpartners, the effective field theory and renormalization group resummation strategy [66-72] for calculating the Higgs mass is probably the best one. One can match the supersymmetric theory onto the standard model parameters as an effective theory at some scale or scales comparable to the most important superpartner masses (probably the top squarks), and then run the parameters of the theory down to a scale comparable to $M_{t}$, and there compute $M_{h}$ within the standard model. In that case, the results obtained here may be a useful ingredient.

\section{ACKNOWLEDGMENTS}

This work was supported in part by the National Science Foundation Grants No. PHY-1068369 and No. PHY1417028. This work was supported in part by the National Science Foundation under Grant No. PHYS1066293 and the hospitality of the Aspen Center for Physics. D. G. R. was supported in part by a grant from the Ohio Supercomputer Center. 


\section{APPENDIX: SOME LOOP INTEGRAL IDENTITIES}

This Appendix contains some loop integral identities that are useful for processing and simplifying the 2-loop Higgs pole mass. Other useful identities in the notation of the present paper can be found in Refs. [38,39,41].

First, the derivatives of 1-loop basis functions, obtained by dimensional analysis and integration by parts, are

$$
\begin{gathered}
\frac{\partial}{\partial x} \mathbf{A}(x)=(d / 2-1) \mathbf{A}(x) / x, \\
\frac{\partial}{\partial x} \mathbf{B}(x, y)=[(d-3)(x-y-s) \mathbf{B}(x, y) \\
+(d-2)\{(x+y-s) \mathbf{A}(x) / 2 x-\mathbf{A}(y)\}] / \Delta_{s x y},
\end{gathered}
$$

where $d=4-2 \epsilon$ is the number of spacetime dimensions and $\Delta_{a b c} \equiv a^{2}+b^{2}+c^{2}-2 a b-2 a c-2 b c$. Using the expansions for small $\epsilon$,

$$
\begin{array}{r}
\mathbf{A}(x)=-x / \epsilon+A(x)+\epsilon A_{\epsilon}(x)+\mathcal{O}\left(\epsilon^{2}\right), \\
\mathbf{B}(x, y)=1 / \epsilon+B(x, y)+\epsilon B_{\epsilon}(x, y)+\mathcal{O}\left(\epsilon^{2}\right),
\end{array}
$$

one then obtains

$$
\begin{gathered}
\frac{\partial}{\partial x} A(x)=A(x) / x+1=\overline{\ln }(x), \\
\frac{\partial}{\partial x} B(x, y)=\{(x-y-s)[B(x, y)-1] \\
+(x+y-s) A(x) / x-2 A(y)]\} / \Delta_{s x y},
\end{gathered}
$$

and the expansions for small $G$ :

$$
\begin{gathered}
A(G)=G \overline{\ln }(G)-G, \\
B(0, G)=B(0,0)+G[3-B(0,0)-\overline{\ln }(G)] / s+\mathcal{O}\left(G^{2}\right),
\end{gathered}
$$

$B(G, G)=B(0,0)+2 G[3-B(0,0)-\overline{\ln }(G)] / s+\mathcal{O}\left(G^{2}\right)$,

$$
\begin{aligned}
B(G, x)= & B(0, x) \\
& +G[3 s-x-(s+x) B(0, x)-2 A(x)] /(x-s)^{2} \\
& +G \overline{\ln }(G) /(x-s)+\mathcal{O}\left(G^{2}\right),
\end{aligned}
$$

$$
\begin{aligned}
A_{\epsilon}(G)= & G\left[-1-\pi^{2} / 12+\overline{\ln }(G)-\frac{1}{2} \overline{\ln }^{2}(G)\right], \quad(\mathrm{A} 11) \\
B_{\epsilon}(0, G)= & B_{\epsilon}(0,0)+G\left[-A_{\epsilon}(G) / G+2 B(0,0)-B_{\epsilon}(0,0)\right] / s \\
+ & \mathcal{O}\left(G^{2}\right), \\
B_{\epsilon}(G, G)= & B_{\epsilon}(0,0) \\
& +2 G\left[-A_{\epsilon}(G) / G+2 B(0,0)-B_{\epsilon}(0,0)\right] / s \\
& +\mathcal{O}\left(G^{2}\right), \\
B_{\epsilon}(G, x)= & B_{\epsilon}(0, x)+G\left[2 A(x)-2 A_{\epsilon}(x)\right. \\
& \left.+(s+x)\left\{2 B(0, x)-B_{\epsilon}(0, x)\right\}\right] /(x-s)^{2} \\
& +A_{\epsilon}(G) /(x-s)+\mathcal{O}\left(G^{2}\right) .
\end{aligned}
$$

Some identities between basis integrals that hold for nongeneric squared mass arguments are the threshold identities:

$$
\begin{gathered}
\lim _{s \rightarrow x} B(0, x)=1-A(x) / x, \\
\lim _{s \rightarrow x}[\bar{T}(0,0, x)+T(x, 0,0)]=-1,
\end{gathered}
$$

and the general relations

$$
\begin{gathered}
I(0,0, x)=A(x)-A(x)^{2} / 2 x-x\left(1+\pi^{2} / 6\right), \\
I(0, x, x)=2 A(x)-A(x)^{2} / x-2 x, \\
\bar{T}(0,0, x)=-T(x, 0,0)+\left[-s+2 A(x)-A(x)^{2} / x\right. \\
+ \\
\left.-\quad s B(0, x)^{2}\right] /(s-x), \\
\bar{T}(0,0,0)=-[B(0,0)-1]^{2} / 2, \\
U(0, x, 0,0)=(1-x / s) T(x, 0,0)+B(0,0) B(0, x) \\
+A(x) B(0,0) / x+(1-x / s) B(0, x) \\
\end{gathered}
$$

Other identities of similar type that express redundancies among the basis integrals for nongeneric squared mass arguments and were used here have appeared as Eqs. (A.14), (A.15), and (A.17)-(A.20) of Ref. [41]. 
[1] G. Aad et al. (ATLAS Collaboration), Phys. Lett. B 716, 1 (2012); S. Chatrchyan et al. (CMS Collaboration), Phys. Lett. B 716, 30 (2012).

[2] G. Aad et al. (ATLAS Collaboration), Phys. Rev. D 90, 052004 (2014); S. Chatrchyan et al. (CMS Collaboration), Report No. CMS-PAS-HIG-13-005.

[3] M. Sher, Phys. Rep. 179, 273 (1989), and references therein.

[4] M. Lindner, M. Sher, and H. W. Zaglauer, Phys. Lett. B 228, 139 (1989).

[5] P. B. Arnold and S. Vokos, Phys. Rev. D 44, 3620 (1991).

[6] C. Ford, D. R. T. Jones, P. W. Stephenson, and M. B. Einhorn, Nucl. Phys. B395, 17 (1993).

[7] J. A. Casas, J. R. Espinosa, and M. Quirós, Phys. Lett. B 342, 171 (1995).

[8] J. R. Espinosa and M. Quiros, Phys. Lett. B 353, 257 (1995).

[9] J. A. Casas, J. R. Espinosa, and M. Quiros, Phys. Lett. B 382, 374 (1996).

[10] G. Isidori, G. Ridolfi, and A. Strumia, Nucl. Phys. B609, 387 (2001).

[11] J. R. Espinosa, G. F. Giudice, and A. Riotto, J. Cosmol. Astropart. Phys. 05 (2008) 002.

[12] N. Arkani-Hamed, S. Dubovsky, L. Senatore, and G. Villadoro, J. High Energy Phys. 03 (2008) 075.

[13] F. Bezrukov and M. Shaposhnikov, J. High Energy Phys. 07 (2009) 089.

[14] J. Ellis, J. R. Espinosa, G. F. Giudice, A. Hoecker, and A. Riotto, Phys. Lett. B 679, 369 (2009).

[15] J. Elias-Miro, J. R. Espinosa, G. F. Giudice, G. Isidori, A. Riotto, and A. Strumia, Phys. Lett. B 709, 222 (2012).

[16] S. Alekhin, A. Djouadi, and S. Moch, Phys. Lett. B 716, 214 (2012).

[17] F. Bezrukov, M. Y. Kalmykov, B. A. Kniehl, and M. Shaposhnikov, J. High Energy Phys. 10 (2012) 140.

[18] G. Degrassi, S. Di Vita, J. Elias-Miro, J. R. Espinosa, G. F. Giudice, G. Isidori, and A. Strumia, J. High Energy Phys. 08 (2012) 098.

[19] D. Buttazzo, G. Degrassi, P. P. Giardino, G. F. Giudice, F. Sala, A. Salvio, and A. Strumia, J. High Energy Phys. 12 (2013) 089.

[20] C. Ford, I. Jack, and D. R. T. Jones, Nucl. Phys. B387, 373 (1992); B504, 551(E) (1997).

[21] S. P. Martin, Phys. Rev. D 89, 013003 (2014).

[22] S. P. Martin, Phys. Rev. D 90, 016013 (2014).

[23] J. Elias-Miro, J. R. Espinosa, and T. Konstandin, J. High Energy Phys. 08 (2014) 034.

[24] R. Tarrach, Nucl. Phys. B183, 384 (1981).

[25] R. G. Stuart, Phys. Lett. B 262, 113 (1991).

[26] A. Sirlin, Phys. Rev. Lett. 67, 2127 (1991).

[27] R. G. Stuart, Phys. Rev. Lett. 70, 3193 (1993).

[28] A. S. Kronfeld, Phys. Rev. D 58, 051501 (1998).

[29] P. Gambino and P. A. Grassi, Phys. Rev. D 62, 076002 (2000).

[30] B. A. Kniehl, Phys. Rev. D 50, 3314 (1994).

[31] A. Djouadi and P. Gambino, Phys. Rev. D 51, 218 (1995); 53, 4111(E) (1996).

[32] M. E. Machacek and M. T. Vaughn, Nucl. Phys. B222, 83 (1983).

[33] M. E. Machacek and M. T. Vaughn, Nucl. Phys. B236, 221 (1984).
[34] I. Jack and H. Osborn, Nucl. Phys. B249, 472 (1985).

[35] M. E. Machacek and M. T. Vaughn, Nucl. Phys. B249, 70 (1985).

[36] O. V. Tarasov, Nucl. Phys. B502, 455 (1997).

[37] R. Mertig and R. Scharf, Comput. Phys. Commun. 111, 265 (1998).

[38] S. P. Martin, Phys. Rev. D 68, 075002 (2003).

[39] S. P. Martin and D. G. Robertson, Comput. Phys. Commun. 174, 133 (2006).

[40] http://www.niu.edu/spmartin/SMH and http://faculty. otterbein.edu/drobertson/SMH.

[41] S. P. Martin, Phys. Rev. D 70, 016005 (2004).

[42] W.-Y. Keung and W. J. Marciano, Phys. Rev. D 30, 248 (1984).

[43] K. G. Chetyrkin and M. F. Zoller, J. High Energy Phys. 06 (2012) 033; 04 (2013) 091.

[44] A. V. Bednyakov, A. F. Pikelner, and V. N. Velizhanin, J. High Energy Phys. 01 (2013) 017; Phys. Lett. B 722, 336 (2013); Nucl. Phys. B875, 552 (2013).

[45] A. Djouadi, J. Kalinowski, and M. Spira, Comput. Phys. Commun. 108, 56 (1998).

[46] D. J. Broadhurst, Z. Phys. C 47, 115 (1990).

[47] R. Scharf and J. B. Tausk, Nucl. Phys. B412, 523 (1994).

[48] A. I. Davydychev and J. B. Tausk, Nucl. Phys. B397, 123 (1993).

[49] A. I. Davydychev, V. A. Smirnov, and J. B. Tausk, Nucl. Phys. B410, 325 (1993).

[50] F. A. Berends and J. B. Tausk, Nucl. Phys. B421, 456 (1994).

[51] M. Caffo, H. Czyz, S. Laporta, and E. Remiddi, Nuovo Cimento A 111, 365 (1998).

[52] S. Dawson, A. Gritsan, H. Logan, J. Qian, C. Tully, R. Van Kooten et al., arXiv:1310.8361, and references therein.

[53] H. E. Haber and R. Hempfling, Phys. Rev. Lett. 66, 1815 (1991).

[54] Y. Okada, M. Yamaguchi, and T. Yanagida, Prog. Theor. Phys. 85, 1 (1991).

[55] J. R. Ellis, G. Ridolfi, and F. Zwirner, Phys. Lett. B 262, 477 (1991).

[56] S. Heinemeyer, W. Hollik, and G. Weiglein, Phys. Rev. D 58, 091701 (1998); Phys. Lett. B 440, 296 (1998); Comput. Phys. Commun. 124, 76 (2000); Eur. Phys. J. C 9, 343 (1999).

[57] S. P. Martin, Phys. Rev. D 71, 016012 (2005).

[58] R. V. Harlander, P. Kant, L. Mihaila, and M. Steinhauser, Phys. Rev. Lett. 100, 191602 (2008); 101, 039901 (2008).

[59] P. Kant, R. V. Harlander, L. Mihaila, and M. Steinhauser, J. High Energy Phys. 08 (2010) 104.

[60] J. S. Lee, M. Carena, J. Ellis, A. Pilaftsis, and C. E. M. Wagner, Comput. Phys. Commun. 184, 1220 (2013).

[61] T. Hahn, S. Heinemeyer, W. Hollik, H. Rzehak, and G. Weiglein, Phys. Rev. Lett. 112, 141801 (2014).

[62] M. S. Carena, M. Quiros, and C. E. M. Wagner, Nucl. Phys. B461, 407 (1996).

[63] J. R. Espinosa and R.-J. Zhang, J. High Energy Phys. 03 (2000) 026; Nucl. Phys. B586, 3 (2000).

[64] S. P. Martin, Phys. Rev. D 66, 096001 (2002).

[65] S. P. Martin, Phys. Rev. D 67, 095012 (2003).

[66] H. E. Haber and R. Hempfling, Phys. Rev. D 48, 4280 (1993). 
[67] J. A. Casas, J. R. Espinosa, M. Quiros, and A. Riotto, Nucl. Phys. B436, 3 (1995); B439, 466(E) (1995).

[68] M. S. Carena, J. R. Espinosa, M. Quiros, and C.E. M. Wagner, Phys. Lett. B 355, 209 (1995).

[69] H. E. Haber, R. Hempfling, and A. H. Hoang, Z. Phys. C 75, 539 (1997).
[70] M. S. Carena, H. E. Haber, S. Heinemeyer, W. Hollik, C. E. M. Wagner, and G. Weiglein, Nucl. Phys. B580, 29 (2000).

[71] S. P. Martin, Phys. Rev. D 75, 055005 (2007).

[72] P. Draper, G. Lee, and C. E. M. Wagner, Phys. Rev. D 89, 055023 (2014). 